\title{
Geodesic $B$-Preinvex Functions and Multiobjective Optimization Problems on Riemannian Manifolds
}

\author{
Sheng-lan Chen, ${ }^{1,2}$ Nan-Jing Huang, ${ }^{1}$ and Donal O'Regan ${ }^{3,4}$ \\ ${ }^{1}$ Department of Mathematics, Sichuan University, Chengdu, Sichuan 610064, China \\ ${ }^{2}$ School of Mathematics and Physics, Chongqing University of Posts and Telecommunications, Chongqing 400065, China \\ ${ }^{3}$ School of Mathematics, Statistics and Applied Mathematics, National University of Ireland, Galway, Ireland \\ ${ }^{4}$ Department of Mathematics, King Abdulaziz University, Jeddah, Saudi Arabia \\ Correspondence should be addressed to Nan-Jing Huang; nanjinghuang@hotmail.com
}

Received 10 January 2014; Accepted 21 February 2014; Published 26 March 2014

Academic Editor: Xian-Jun Long

Copyright (c) 2014 Sheng-lan Chen et al. This is an open access article distributed under the Creative Commons Attribution License, which permits unrestricted use, distribution, and reproduction in any medium, provided the original work is properly cited.

\begin{abstract}
We introduce a class of functions called geodesic $B$-preinvex and geodesic $B$-invex functions on Riemannian manifolds and generalize the notions to the so-called geodesic quasi/pseudo $B$-preinvex and geodesic quasi/pseudo $B$-invex functions. We discuss the links among these functions under appropriate conditions and obtain results concerning extremum points of a nonsmooth geodesic $B$-preinvex function by using the proximal subdifferential. Moreover, we study a differentiable multiobjective optimization problem involving new classes of generalized geodesic $B$-invex functions and derive Kuhn-Tucker-type sufficient conditions for a feasible point to be an efficient or properly efficient solution. Finally, a Mond-Weir type duality is formulated and some duality results are given for the pair of primal and dual programming.
\end{abstract}

\section{Introduction}

Convex functions play an important role in optimization theory and there are several classes of functions given in the literature with the goal to weaken the limitations of convexity in mathematical programming. Generalized convex functions, labelled as $B$-vex functions, were introduced by Bector and Singh [1]. In 1981, Hanson [2] introduced the concept of invexity and proved that the Kuhn-Tucker conditions are sufficient for optimality of a nonlinear programming problem under invexity conditions. Preinvex functions were defined by Ben-Israel and Mond [3], and, in [4], Weir and Mond showed how and where preinvex functions could replace convex functions in multiple objective optimization problem. These functions were further generalized to pseudo/quasi $B$ vex, $B$-invex, and pseudo/quasi $B$-invex functions by Bector et al. [5] and to $B$-preinvex by Suneja et al. [6]. In [5], Bector et al. obtained sufficient optimality criteria and duality results for a nonlinear programming problem involving $B$-vex and $B$-invex functions. There are also many papers in the literature concerning the generalization of convexity in connection with sufficiency and duality in optimization problems (see, e.g., $[7-12]$ and the references therein).
A manifold is not a linear space and extensions of concepts and techniques from linear spaces to Riemannian manifolds are natural. In the literature many authors studied generalized convex functions and many results in convex analysis and optimization theory were extended to Riemannian manifolds (see [13-28] and the references therein). Rapcsák [27] and Udriste [28] considered a generalization of convexity called geodesic convexity. In this setting the linear space is replaced by a Riemannian manifold and the line segment by a geodesic. Pini [22] introduced the notion of invex function on Riemannian manifolds, while Mititelu [24] investigated its generalization. The concepts of geodesic invex sets, geodesic invex, and preinvex functions on Riemannian manifolds were defined by Barani and Pouryayevali [17]. They established the relation between geodesic invexity and preinvexity of functions, and they also obtained results concerning extremum points of a nonsmooth geodesic preinvex function by using the proximal subdifferential. Subsequently, Agarwal et al. [20] proposed and discussed geodesic $\alpha$ preinvexity on Riemannian manifolds, which generalized the corresponding results studied by Barani and Pouryayevali [17]. A new concept of geodesic roughly $B$-invexity and its generalization on Hadamard manifolds were introduced 
by Zhou and Huang [26]. They studied the properties of these functions and they established sufficient optimality conditions and duality in nonlinear programming problems.

In this paper, we introduce a class of geodesic $B$-preinvex and $B$-invex functions on Riemannian manifolds and extend them to geodesic quasi/pseudo $B$-preinvex and geodesic quasi/pseudo $B$-invex functions. We discuss the links among these functions under suitable assumptions. By applying the proximal subdifferential, we relax the smoothness condition and study the question of global minima for geodesic $B$ preinvex functions on Riemannian manifolds. As applications, we investigate a multiobjective programming problem involving generalized geodesic $B$-invex functions and derive the Kuhn-Tucker-type sufficient optimality conditions for a feasible point to be an efficient or properly efficient solution. Finally, a Mond-Weir type duality is formulated and some duality results are obtained for the pair of primal and dual programming. The results presented in this paper extend some known results due to Barani and Pouryayevali $[17,23]$.

\section{Preliminaries}

In this section, we recall some definitions and known results about Riemannian manifolds which will be used throughout the paper. These can be found in many introductory books on Riemannian geometry, such as in [29-32].

Let $M$ be a $C^{\infty}$ smooth manifold modelled on a Hilbert space $H$, either finite dimensional or infinite dimensional, endowed with a Riemannian metric $\langle\cdot, \cdot\rangle_{p}$ on the tangent space $T_{p} M \cong H$. The corresponding norm is denoted by $\|\cdot\|_{p}$. The tangent bundle of $M$ is denoted by $T M=$ $\bigcup_{x \in M} T_{x} M$, which is naturally a manifold. Given a piecewise $C^{1}$ path $\gamma:[a, b] \rightarrow M$ joining $p$ to $q$, that is, $\gamma(a)=p$ and $\gamma(b)=q$, we can define the length of $\gamma$ by

$$
L(\gamma):=\int_{a}^{b}\left\|\gamma^{\prime}(t)\right\|_{\gamma(t)} d t .
$$

For any two points $p, q \in M$, we define

$$
\begin{aligned}
& d(p, q) \\
& \quad=\inf \left\{L(\gamma): \gamma \text { is a piecewise } C^{1} \text { path joining } p \text { to } q\right\} .
\end{aligned}
$$

Then $d$ is a metric on $M$ which defines the same topology as the one $M$ naturally has as a manifold. For this metric we define the open ball centered at the point $p$ with radius $r>0$; that is,

$$
B(p, r)=\{q \in M: d(p, q)<r\} .
$$

Let us recall that in every Riemannian manifold there exists exactly one covariant derivation called the Levi-Civita connection denoted by $\nabla_{X} Y$ for any vector fields $X, Y$ on $M$. We also recall that a geodesic is a $C^{\infty}$ smooth path $\gamma$ whose tangent is parallel along the path $\gamma$; that is, $\gamma$ satisfies the equation $\nabla_{d \gamma(t) / d t} d \gamma(t) / d t=0$. Any path $\gamma$ joins $p$ and $q$ in $M$ such that $L(\gamma)=d(p, q)$ is a geodesic and is called a minimal geodesic. The existence theorem for ordinary differential equation implies that for every $v \in T M$, there exists an open interval $J(v)$ containing 0 and exactly one geodesic $\gamma(v)$ : $J(v) \rightarrow M$ with $\gamma_{v}^{\prime}(0)=v$. This implies that there is an open neighborhood $\widetilde{T} M$ of the submanifold $M$ of $T M$ such that for every $v \in \widetilde{T} M$, the geodesic $\gamma_{v}(t)$ is defined for $|t|<2$ (see, e.g., [25]). The exponential map $\exp _{p}: T_{p} M \subseteq \widetilde{T} M \rightarrow M$ is then defined as $\exp _{p}(v)=\gamma_{v}(1)$, where $\gamma_{v}$ is the geodesic defined by its position $p$ and velocity $\gamma_{v}^{\prime}(0)=v$ at $p$.

If $\gamma$ is a geodesic, then for each $t_{1}, t_{2} \in[a, b]$, the LeviCivita connection $\nabla$ induces an isometry $P_{t_{1}, \gamma}^{t_{2}}: T_{\gamma\left(t_{1}\right)} M \rightarrow$ $T_{\gamma\left(t_{2}\right)} M$, the so-called parallel translation from $T_{\gamma\left(t_{1}\right) M}$ to $T_{\gamma\left(t_{2}\right)} M$ along $\gamma$, which is defined by

$$
P_{t_{1}, \gamma}^{t_{2}}(v)=V\left(\gamma\left(t_{2}\right)\right) \quad \forall a, b \in R, v \in T_{\gamma\left(t_{1}\right)} M,
$$

where $V$ is the unique vector field satisfying $\nabla_{d \gamma(t) / d t} V=0$ for all $t$ and $V\left(\gamma\left(t_{1}\right)\right)=v$.

Let $f: M \rightarrow R$ be a differentiable function. We will denote by

$$
d f_{x}: T_{p} M \longrightarrow T_{f(x)} R \equiv R
$$

the differential $f$ at $x$.

We also recall that a simply connected complete Riemannian manifold of nonpositive curvature is called a CartanHadamard manifold.

Barani and Pouryayevali [17] first defined geodesic invex sets and introduced geodesic preinvex functions on Riemannian manifolds.

Definition 1. Let $M$ be a Riemannian manifold and $\eta: M \times$ $M \rightarrow T M$ be a function such that $\eta(x, y) \in T_{y} M$ for every $x, y \in M$. A nonempty subset $S$ of $M$ is said to be geodesic invex with respect to $\eta$, if for every $x, y \in S$, there exists exactly one geodesic $\gamma_{x, y}:[0,1] \rightarrow M$ such that

$$
\begin{aligned}
& \gamma_{x, y}(0)=y, \quad \gamma_{x, y}^{\prime}(0)=\eta(x, y), \\
& \gamma_{x, y}(t) \in S, \quad \forall t \in[0,1] .
\end{aligned}
$$

Definition 2. Let $M$ be a Riemannian manifold and $S \subseteq M$ a geodesic invex set with respect to $\eta: M \times M \rightarrow T M$. We say that a function $f: S \rightarrow R$ is geodesic preinvex if

$$
\begin{array}{r}
f\left(\gamma_{x, y}(t)\right) \leqq \\
\forall f(x)+(1-t) f(y), \\
\forall x, y \in S, \quad t \in[0,1] .
\end{array}
$$

In 1993, Suneja et al. [6] introduced the generalization of preinvex functions on $R^{n}$, and we now improve and extend the definition of $B$-preinvex functions to Riemannian manifolds.

Definition 3. Let $M$ be a Riemannian manifold and $S \subseteq M$ an open invex set with respect to $\eta: M \times M \rightarrow T M$. A function $f: S \rightarrow R$ is said to be 
(i) geodesic $B$-preinvex (GBPIX) at $y \in S$ with respect to $\eta$ and $b$ if

$$
\begin{array}{r}
f\left(\gamma_{x, y}(t)\right) \leqq t b(x, y, t) f(x)+(1-t b(x, y, t)) f(y), \\
\forall x \in S, \quad t \in[0,1],
\end{array}
$$

where $b: S \times S \times[0,1] \rightarrow R_{+}$with $t b(x, y, t) \in[0,1]$ for all $x, y \in S$ and $t \in[0,1]$, and $\gamma_{x, y}$ is the unique geodesic defined in Definition 1;

(ii) GBPIX on $S$ with respect to $\eta$ and $b$ if it is GBPIX at each $y \in S$ with respect to the same $\eta$ and $b$;

(iii) strictly GBPIX (SGBPIX) on $S$ with respect to $\eta$ and $b$ if inequality (8) is strict for all $x, y \in S$ with $x \neq y$;

(iv) geodesic quasi $B$-preinvex (GQBPIX) with respect to $\eta$ and $b$ at $y \in S$ if

$$
\begin{array}{r}
f(x) \leqq f(y) \Longrightarrow b(x, y, t)\left(f\left(\gamma_{x, y}(t)\right)-f(y)\right) \leqq 0, \\
\forall x \in S, \quad t \in[0,1] ;
\end{array}
$$

(v) GQBPIX on $S$ with respect to $\eta$ and $b$ if it is GQBPIX at each $y \in S$ with respect to the same $\eta$ and $b$;

(vi) geodesic pseudo $B$-preinvex (GPBPIX) with respect to $\eta$ and $b$ if, there exists a strictly positive function $a: S \times S \rightarrow R$ such that

$$
\begin{aligned}
b(x, y, t) f(x) & \leqq b(x, y, t) f(y) \Longrightarrow f\left(\gamma_{x, y}(t)\right)-f(y) \\
& \leqq t(t-1) a(x, y), \quad \forall x \in S, t \in[0,1]
\end{aligned}
$$

(vii) GPBPIX on $S$ with respect to $\eta$ and $b$, if it is GPBPIX at each $y \in S$ with respect to the same $\eta$ and $b$.

Remark 4. By Definition 1, it is clear that SGBPIX $\Rightarrow$ GBPIX, GBPIX $\Rightarrow$ GQBPIX, and GPBPIX $\Rightarrow$ GQBPIX.

Remark 5. If $b>0$, then GBPIX $\Rightarrow$ GPBPIX.

Remark 6. The above definition of geodesic $B$-preinvexity on Riemannian manifolds is also a generalization of geodesic preinvexity discussed in [17]. It is easy to see that every geodesic preinvex function $f$ with respect to $\eta$ is a geodesic $B$ preinvex function with respect to $\eta$ and $b$, where $b(x, y, t)=1$, but the converse is not true, as illustrated in the following example.

Example 7. Let $M=\left\{e^{i \theta} \mid 0 \leq \theta \leq \pi / 2\right\}$. For any $x, y \in M$ with $x=e^{i \theta}$ and $y=e^{i \phi}$, let $f: M \rightarrow R$ be defined by $f(x)=\sin \theta$, let $\eta: M \times M \rightarrow T M$ be defined by

$$
\eta\left(e^{i \theta}, e^{i \phi}\right)=(\phi-\theta)(\sin \phi,-\cos \phi),
$$

and let $b: M \times M \times[0,1] \rightarrow R_{+}$be defined by

$$
b\left(e^{i \theta}, e^{i \phi}, t\right)= \begin{cases}\frac{1}{t}, & \theta \geq \phi, \\ 0, & \theta<\phi .\end{cases}
$$

Define a geodesic $\gamma_{x, y}(t)$ on $M$ as follows:

$$
\gamma_{x, y}(t)=(\cos ((1-t) \phi+t \theta), \sin ((1-t) \phi+t \theta)) .
$$

Then, we can easily verify that $f$ is geodesic $B$-preinvex with respect to $\eta$ and $b$, but $f$ is not geodesic preinvex with respect to $\eta$.

In [22], Pini introduced the concept of geodesic invexity on Riemannian manifolds. Motivated by the definitions of (pseudo/quasi) $B$-invex functions on $R^{n}$ given in [5], we present the following definition.

Definition 8. Let $M$ be a Riemannian manifold and $b: M \times$ $M \rightarrow R_{+}$a nonnegative real function. A differentiable function $f: M \rightarrow R$ is said to be

(i) geodesic $B$-invex (GBIX) at $y \in M$ with respect to $\eta$ and $b$ if

$d f_{y}(\eta(x, y)) \leqq b(x, y)(f(x)-f(y)), \quad \forall x \in M ;$

(ii) GBIX on $M$ with respect to $\eta$ and $b$ if it is GBIX at each $y \in M$ with respect to the same $\eta$ and $b$;

(iii) strictly geodesic $B$-invex on $M$ with respect to $\eta$ and $b$ if inequality (14) is strict for all $x, y \in M$ with $x \neq y$;

(iv) geodesic quasi $B$-invex (GQBIX) at $y \in M$ with respect to $\eta$ and $b$ if

$f(x) \leqq f(y) \Longrightarrow b(x, y) d f_{y}(\eta(x, y)) \leqq 0, \quad \forall x \in M$

(v) GQBIX on $M$ with respect to $\eta$ and $b$ if it is GQBIX at each $y \in M$ with respect to the same $\eta$ and $b$;

(vi) geodesic pseudo $B$-invex (GPBIX) at $y \in M$ with respect to $\eta$ and $b$ if

$$
\begin{aligned}
d f_{y}(\eta(x, y)) & \geqq 0 \Longrightarrow b(x, y) f(x) \\
& \geqq b(x, y) f(y), \quad \forall x \in M,
\end{aligned}
$$

or equivalently,

$$
\begin{aligned}
& b(x, y) f(x) \\
& <b(x, y) f(y) \Longrightarrow d f_{y}(\eta(x, y))<0, \quad \forall x \in M
\end{aligned}
$$

(vii) strictly geodesic pseudo $B$-invex (SGPBIX) at $y \in M$ with respect to $\eta$ and $b$ if

$$
\begin{array}{r}
d f_{y}(\eta(x, y)) \geqq 0 \Longrightarrow b(x, y) f(x)>b(x, y) f(y), \\
\forall x \in M, \quad x \neq y,
\end{array}
$$

or equivalently,

$$
\begin{array}{r}
b(x, y) f(x) \leqq b(x, y) f(y) \Longrightarrow d f_{y}(\eta(x, y))<0, \\
\forall x \in M, \quad x \neq y ;
\end{array}
$$


(viii) GPBIX/SGBPIX on $M$ with respect to $\eta$ and $b$ if it is GPBIX/SGBPIX at each $y \in M$ with respect to the same $\eta$ and $b$.

Remark 9. An invex function $f$ with respect to $\eta$ discussed on Riemannian manifolds in [17] is also a GBIX function with respect to $\eta$ and $b$ with $b=1$, but the converse is not true.

Example 10. Let $M$ be a Riemannian manifold and $f: M \rightarrow$ $R$ a differentiable function such that for every $y \in M, d f_{y} \neq 0$. Let $b: M \times M \rightarrow R_{+}$be a bifunction and let $\eta: M \times M \rightarrow$ $T M$ be defined by

$$
\eta(x, y)=\frac{b(x, y)(f(x)-f(y))}{\left\|d f_{y}\right\|_{y}^{2}} d f_{y} .
$$

Then, for every $x, y \in M$, one has

$$
\begin{aligned}
\left\langle d f_{y}, \eta(x, y)\right\rangle_{y} & =b(x, y)\left\langle d f_{y}, \frac{f(x)-f(y)}{\left\|d f_{y}\right\|_{y}^{2}} d f_{y}\right\rangle_{y} \\
& =b(x, y) \frac{f(x)-f(y)}{\left\|d f_{y}\right\|^{2}}\left\langle d f_{y}, d f_{y}\right\rangle_{y} \\
& =b(x, y)[f(x)-f(y)] .
\end{aligned}
$$

Therefore, $f$ is geodesic $B$-invex with respect to $\eta$ and $b$, but $f$ is not geodesic invex with respect to the same $\eta$ whenever $b(x, y) \neq 1$.

Remark 11. Every geodesic $B$-invex function $f$ with respect to $\eta$ and $b$, where $b(x, y)>0, \forall x, y \in M$, is geodesic invex with respect to some $\bar{\eta}$, where

$$
\bar{\eta}(x, y)=\frac{\eta(x, y)}{b(x, y)} .
$$

Remark 12. Every geodesic pseudoinvex function with respect to $\eta$ in [18] is geodesic pseudo $B$-invex with respect to the same $\eta$. However, the converse is not necessarily true when $b(x, y)=0$, for some $x, y \in M$.

Remark 13. From Definition 8, we have

$$
\begin{gathered}
\text { SGBIX } \Longrightarrow \text { GBIX } \Longrightarrow \text { GBPIX } \\
\Downarrow
\end{gathered}
$$

\section{GBQIX,}

Finally we present the following definitions which will be useful in the sequel.

Definition 14 (see [17]). Let $M$ be a Riemannian manifold. We say that the function $\eta: M \times M \rightarrow T M$ satisfies the condition $(C)$, if for each $x, y \in M$, and for the geodesic $\gamma:[0,1] \rightarrow M$ satisfying $\gamma_{x, y}(0)=y, \gamma^{\prime}(0)=\eta(x, y)$, we have

(i) $P_{t, \gamma}^{0}\left[\eta\left(y, \gamma_{x, y}(t)\right)\right]=-t \eta(x, y)$;

(ii) $P_{t, \gamma}^{0}\left[\eta\left(x, \gamma_{x, y}(t)\right)\right]=(1-t) \eta(x, y)$.
Definition 15 (see [33]). Let $M$ be a Riemannian manifold and $f: M \rightarrow(-\infty,+\infty)$ a lower semicontinuous function. A point $\zeta \in T_{y} M$ is a proximal subgradient of $f$ at $y \in \operatorname{dom}(f)$ if there exist positive numbers $\delta$ and $\sigma$ such that

$$
f(x) \geq f(y)+\left\langle\zeta, \exp _{y}^{-1} x\right\rangle_{y}-\sigma d(y, x)^{2}, \quad \forall x \in B(y, \delta),
$$

where $\operatorname{dom}(f)=\{x \in M: f(x)<\infty\}$.

The set of all proximal subgradients of $f$ at $y \in M$ is denoted by $\partial_{p} f(y)$ and is called the proximal subdifferential of $f$ at $y$.

\section{GBPIX (GBIX) Functions and Their Generalization}

Theorem 16. Let $M$ be a Riemannian manifold, let $S$ be an open invex subset of $M$ with respect to $\eta: M \times M \rightarrow T M$, and let $b: S \times S \times[0,1] \rightarrow R_{+}$be a nonnegative real function. Assume that $f: S \rightarrow R$ is a differentiable GBPIX function with respect to $\eta$ and $b$. Then $f$ is a GBIX function with respect to $\eta$ and $\bar{b}$, where $\bar{b}(x, y)=\lim _{t \rightarrow 0^{+}} b(x, y, t)$.

Proof. Let $x, y \in S$. Since $S$ is a geodesic invex set with respect to $\eta$, there exists exactly one geodesic $\gamma_{x, y}:[0,1] \rightarrow M$ such that

$$
\begin{aligned}
& \gamma_{x, y}(0)=y, \quad \gamma_{x, y}^{\prime}(0)=\eta(x, y), \\
& \gamma_{x, y}(t) \in S, \quad \forall t \in[0,1] .
\end{aligned}
$$

Noting that $f$ is GBPIX with respect to $\eta$ and $b$, we have

$$
f\left(\gamma_{x, y}(t)\right) \leqq t b f(x)+(1-t b) f(y), \quad \forall t \in(0,1),
$$

which implies

$$
f\left(\gamma_{x, y}(t)\right)-f(y) \leqq t b(x, y, t)(f(x)-f(y)) .
$$

Dividing by $t$ and taking the limit as $t \rightarrow 0$, we obtain

$$
d f_{\gamma_{x, y}(0)}\left(\gamma_{x, y}^{\prime}(0)\right) \leqq \bar{b}(x, y)(f(x)-f(y)) .
$$

Therefore, $d f_{\gamma_{x, y}(0)}(\eta(x, y)) \leqq \bar{b}(x, y)(f(x)-f(y))$. This completes the proof.

Theorem 17. Let $M$ be a Riemannian manifold and $S \subseteq M$ an open geodesic invex set with respect to $\eta: M \times M \rightarrow T M$. Suppose that $f: S \rightarrow R$ is differentiable GBIX with respect to $\eta$ and $b$, where $b: S \times S \rightarrow R_{+}$. If $\eta$ satisfies condition $(C)$ and $b(x, y)>0$, for all $x, y \in S$, then $f$ is GBPIX with respect to $\eta$ and $\bar{b}$, where

$$
\bar{b}(x, y, t)=\frac{b(x, \bar{x})}{(1-t) b(y, \bar{x})+t b(x, \bar{x})}
$$

for all $x, y \in S$ and $\bar{x}=\gamma_{x, y}(t)$, for some $t \in[0,1]$. 
Proof. Since $S$ is geodesic invex with respect to $\eta$, there exists exactly one geodesic $\gamma_{x, y}:[0,1] \rightarrow M$ such that

$$
\begin{aligned}
& \gamma_{x, y}(0)=y, \quad \gamma_{x, y}^{\prime}(0)=\eta(x, y), \quad \gamma_{x, y}(t) \in S, \\
& \forall x, y \in S, \quad \forall x, y \in[0,1] .
\end{aligned}
$$

Fix $t \in[0,1]$ and set $\bar{x}=\gamma_{x, y}(t)$. Then we have

$$
\begin{aligned}
& b(x, \bar{x})(f(x)-f(\bar{x})) \geqq d f_{\bar{x}}(\eta(x, \bar{x})), \\
& b(y, \bar{x})(f(y)-f(\bar{x})) \geqq d f_{\bar{x}}(\eta(y, \bar{x})) .
\end{aligned}
$$

Now multiplying (31) and (32) by $t$ and ( $1-t)$, respectively, and adding, then we have

$$
\begin{array}{r}
t b(x, \bar{x}) f(x)+(1-t) b(y, \bar{x}) f(y) \\
-(t b(x, \bar{x})+(1-t) b(y, \bar{x})) f(\bar{x}) \\
\geqq d f_{\bar{x}}(t \eta(x, \bar{x})+(1-t) \eta(y, \bar{x})) .
\end{array}
$$

It follows from condition $(C)$ that

$$
\begin{aligned}
t \eta(x, \bar{x}) & +(1-t) \eta(y, \bar{x}) \\
= & t(1-t) P_{0, \gamma_{x, y}}^{t}[\eta(x, y)] \\
& +(1-t)(-t) P_{0, \gamma_{x, y}}^{t}[\eta(x, y)]=0 .
\end{aligned}
$$

Therefore,

$$
f(\bar{x}) \leqq t \bar{b}(x, y, t) f(x)+(1-t \bar{b}(x, y, t)) f(y),
$$

where

$$
\bar{b}(x, y, t)=\frac{b(x, \bar{x})}{(1-t) b(y, \bar{x})+t b(x, \bar{x})} .
$$

This completes the proof.

Theorem 18. Let $M$ be a Riemannian manifold, let $S \subseteq M$ be an open geodesic invex set with respect to $\eta$, and let $f: S \rightarrow R$ be GBPIX with respect to $\eta$ and $b$.

(i) Every lower section of $f$ defined by

$$
L(f, \lambda)=\{x \in S \mid f(x) \leqq \lambda\}, \quad \lambda \in R,
$$

is a geodesic invex set with respect to $\eta$.

(ii) The set $K$ of solutions for problem

$$
\begin{array}{ll}
(P) \quad \min & f(x) \\
\text { s.t. } & x \in S
\end{array}
$$

is a geodesic invex set with respect to $\eta$.

(iii) If $\bar{x} \in S$ is a local optimal solution to the problem $(P)$ and $b>0$, then $\bar{x}$ is a global minimum for $(P)$. Moreover, if $f$ is strictly GBPIX, then the global optimal solution of problem $(P)$ is unique.
Proof. (i) Let $x, y \in L_{f}(\lambda)$. Since $S$ is a geodesic invex set with respect to $\eta$, there exists exactly one geodesic $\gamma_{x, y}:[0,1] \rightarrow$ $M$ such that

$$
\begin{array}{r}
\gamma_{x, y}(0)=y, \quad \gamma_{x, y}^{\prime}(0)=\eta(x, y), \\
\gamma_{x, y}(t) \in S, \quad \forall t \in[0,1] .
\end{array}
$$

The GBPIX of $f$ gives

$$
f\left(\gamma_{x, y}(t)\right) \leqq t b f(x)+(1-t b) f(y) \leqq \lambda, \quad \forall t \in[0,1]
$$

which implies that $\gamma_{x, y}(t) \in L(f, \lambda)$ for all $t \in[0,1]$.

(ii) If $f$ has no optimal solution in $S$, then $K=\emptyset$, which is obviously a geodesic invex set. If $K \neq \emptyset$ and $\bar{x}$ is an arbitrary optimal point for $(P)$, then $K=S \cap L_{f}(f(\bar{x}))$, which is also a geodesic invex set with respect to $\eta$ by $(i)$.

(iii) Suppose that $\bar{x} \in S$ is a local minimum. Then there is a neighborhood $N_{\varepsilon}(\bar{x})$ such that

$$
f(\bar{x}) \leqq f(x), \quad x \in S \cap N_{\varepsilon}(\bar{x}) .
$$

If $\bar{x}$ is not a global minimum of $f$, then there exists a point $x^{*} \in S$ such that

$$
f\left(x^{*}\right)<f(\bar{x}) .
$$

Since $S$ is a geodesic invex set with respect to $\eta$, there exists exactly one geodesic $\gamma_{x^{*}, \bar{x}}$ such that

$$
\begin{array}{r}
\gamma_{x^{*}, \bar{x}}(0)=\bar{x}, \quad \gamma_{x^{*}, \bar{x}}^{\prime}(0)=\eta\left(x^{*}, \bar{x}\right), \\
x^{*}, \bar{x}(t) \in S, \quad \forall t \in[0,1] .
\end{array}
$$

By the continuity of the distance function $d$ and the geodesic $\gamma_{x^{*}, \bar{x}}$, there exists a number $\delta>0$ such that $d\left(\gamma_{x^{*}, \bar{x}}(t), \bar{x}\right)<\varepsilon$ for all $t \in(0, \delta)$. Hence, $\gamma_{x^{*}, \bar{x}}(t) \in N_{\varepsilon}(\bar{x})$. It follows from the GBPIX of $f$ that

$$
\begin{array}{r}
f\left(\gamma_{x^{*}, \bar{x}}(t)\right) \leqq t b f\left(x^{*}\right)+(1-t b) f(\bar{x})<f(\bar{x}), \\
\forall t \in(0,1) .
\end{array}
$$

Hence, for each $\gamma_{x^{*}, \bar{x}}(t) \in S \cap N_{\varepsilon}(\bar{x}), f\left(\gamma_{x^{*}, \bar{x}}(t)\right)<f(\bar{x})$, which is a contradiction to (41).

If $x^{0}$ is another global optimal solution for $(P)$ and $x^{0} \neq \bar{x}$, then $f\left(x^{0}\right)=f(\bar{x})$. It follows from the strict GBPIX of $f$ that

$$
f\left(\gamma_{x^{0}, \bar{x}}(t)\right)<t b f(\bar{x})+(1-t b) f\left(x^{0}\right)=f(\bar{x}),
$$

which contradicts the optimality of $\bar{x}$ for $(P)$. Therefore, the solution of $(P)$ is unique. This completes the proof.

Similar reasoning to that in the proof of Theorem 5.2 in [17] yields the following result.

Theorem 19. Let $M$ be a Cartan-Hadamard manifold and $S \subseteq M$ be an open geodesic invex set with respect to $\eta$ : $M \times M \rightarrow T M$ with $\eta(x, y) \neq 0$ for $x \neq y$. Suppose that $f:$ $S \rightarrow(-\infty,+\infty]$ is a lower semicontinuous GBPIX function 
with respect to $\eta$ and $b$. Let $y \in \operatorname{dom}(f)$ and $\zeta \in \partial_{p} f(y)$. Then there exists a number $\delta>0$ such that

$$
\bar{b}(x, y)[f(x)-f(y)] \geqq\langle\zeta, \eta(x, y)\rangle_{y}, \quad \forall x \in B(y, \delta),
$$

where $\bar{b}(x, y)=\lim _{t \rightarrow 0^{+}} b(x, y, t)$.

From Theorem 19, we can obtain the following corollary.

Corollary 20. Let $M$ be a Cartan-Hadamard manifold and $S \subseteq M$ be an open geodesic invex set with respect to $\eta: M \times$ $M \rightarrow T M$ with $\eta(x, y) \neq 0$ for $x \neq y$. Suppose that $f: S \rightarrow$ $(-\infty,+\infty]$ is a lower semicontinuous geodesic B-preinvex function with respect to $\eta$ and $b$ and $\lim _{t \rightarrow 0^{+}} b(x, y, t)=$ $\bar{b}(x, y)>0$. Let $y \in \operatorname{dom}(f)$ and $0 \in \partial_{p} f(y)$. Then $y$ is a global minimum of $f$.

Remark 21. It should be noted that if $S$ is a subset of a Riemannian manifold $M$ and $f: S \rightarrow(-\infty,+\infty)$ is a lower semicontinuous function which has a local minimum at $y \in$ $S$, then $0 \in \partial_{p} f(y)($ see $[33])$.

Remark 22. Theorems 16, 17, 18, and 19 extend not only the corresponding results from $R^{n}$ to Riemannian manifolds, but also Theorems 4.1, 4.2, 5.1, and 5.2 of [17], respectively.

The following theorems reveal the relations among geodesic quasi $B$-preinvexity, geodesic quasi $B$-invexity, geodesic pseudo $B$-preinvexity, and geodesic pseudo $B$-invexity for a differentiable function $f$.

Theorem 23. Let $M$ be a Riemannian manifold and $S \subseteq M$ an open geodesic invex set with respect to $\eta$. Assume that $f$ : $S \rightarrow(-\infty,+\infty]$ is differentiable GQBPIX with respect to $\eta$ and $b$. Then $f$ is GQBIX with respect to $\eta$ and $\bar{b}$, where $\bar{b}(x, y)=$ $\lim _{t \rightarrow 0^{+}} b(x, y, t)$.

Proof. Let $x, y \in S$ and $f(x) \leqq f(y)$. Since $f$ is GQBPIX with respect to $\eta$ and $b$, we have

$$
b(x, y, t)\left(f\left(\gamma_{x, y}(t)\right)-f(y)\right) \leqq 0, \quad \forall t \in(0,1] .
$$

Dividing the above inequality by $t$ and letting $t \rightarrow 0$, we get

$$
\bar{b}(x, y) d f_{y}(\eta(x, y)) \leqq 0 \quad \forall x, y \in S,
$$

which shows that $f$ is GQBIX with respect to $\eta$ and $\bar{b}$. This completes the proof.

Theorem 24. Let $M$ be a Riemannian manifold and $S \subseteq M$ an open geodesic invex set with respect to $\eta$. Suppose that $\eta$ satisfies condition $(C)$ and $b: M \times M \rightarrow R_{+}$is continuous with respect to the second argument. If $f$ is differentiable GQBIX with respect to $\eta$ and $b$, then $f$ is GQBPIX with respect to $\eta$ and $\bar{b}$, where $\bar{b}(x, y, t)=b(x, \widehat{x}) b(y, \widehat{x})$ for all $x, y \in S$ and $\widehat{x}=\gamma_{x, y}(t)$ for some $t \in[0,1]$.
Proof. Let $x, y \in S$. Since $S$ is a geodesic invex set with respect to $\eta$, there exists exactly one geodesic $\gamma_{x, y}:[0,1] \rightarrow M$ such that

$$
\begin{array}{r}
\gamma_{x, y}(0)=y, \quad \gamma_{x, y}^{\prime}(0)=\eta(x, y), \\
\gamma_{x, y}(t) \in S, \quad \forall t \in[0,1] .
\end{array}
$$

Let $f(x) \leq f(y)$. Consider the set

$$
\begin{aligned}
\Omega=\{z \mid & z=\gamma_{x, y}(t), \\
& \bar{b}(x, y, t) f\left(\gamma_{x, y}(t)\right) \\
& >\bar{b}(x, y, t) f(y), t \in[0,1]\} .
\end{aligned}
$$

In order to show that $f$ is GQBPIX, we have to show that $\Omega=\emptyset$. It is evident that $\Omega$ is equivalent to the set

$$
\begin{gathered}
\Omega^{\prime}=\left\{z \mid z=\gamma_{x, y}(t), f\left(\gamma_{x, y}(t)\right)>f(y),\right. \\
\bar{b}(x, y, t)>0, t \in[0,1]\} .
\end{gathered}
$$

If $\Omega^{\prime} \neq \emptyset$, then, by the continuity of $f$ and $b$, the set

$$
\begin{gathered}
\Omega^{\prime \prime}=\left\{z \mid z=\gamma_{x, y}(t), f\left(\gamma_{x, y}(t)\right)>f(y),\right. \\
\bar{b}(x, y, t)>0, t \in(0,1)\}
\end{gathered}
$$

is also nonempty. Hence, it is sufficient to show that $\Omega^{\prime \prime}=\emptyset$, to complete the proof.

Suppose now that $\bar{x} \in \Omega^{\prime \prime}$. We then have $\bar{x}=\gamma_{x, y}(\bar{t})$, for some $0<\bar{t}<1, f(\bar{x})>f(y) \geqq f(x)$, and $\bar{b}(x, y, \bar{t})>0$. By the definition of GQBIX of, it follows, considering the pair $\bar{x}$ and $x$, that

$$
b(x, \bar{x}) d f_{\bar{x}}(\eta(x, \bar{x})) \leqq 0 .
$$

Similarly, considering the pair $\bar{x}$ and $y$, it follows that

$$
b(y, \bar{x}) d f_{\bar{x}}(\eta(y, \bar{x})) \leqq 0 .
$$

Hence by condition $(C)$, we have

$$
\begin{array}{r}
(1-\bar{t}) b(x, \bar{x}) d f_{\bar{x}} P_{0, \gamma}^{\bar{t}}[\eta(x, y)] \leqq 0, \\
-\bar{t} b(y, \bar{x}) d f_{\bar{x}} P_{0, \gamma}^{\bar{t}}[\eta(x, y)] \leqq 0 .
\end{array}
$$

Now (55), together with the fact that $0<\bar{t}<1$, imply that

$$
\begin{aligned}
& b(x, \bar{x}) b(y, \bar{x}) d f_{\bar{x}} P_{0, \gamma}^{\bar{t}}[\eta(x, y)] \\
& =b(x, \bar{x}) b(y, \bar{x}) d f_{\bar{x}} \gamma_{x, y}^{\prime}(\bar{t})=0 .
\end{aligned}
$$

Note that (56) holds for any $\bar{x} \in \Omega^{\prime \prime}$. Now suppose that $\Omega^{\prime \prime} \neq \emptyset$. Let $\bar{x} \in \Omega^{\prime \prime}$ and let $\bar{x}=\gamma_{x, y}(\bar{t})$. By the continuity of $f, \gamma$, and $b$, we can find $0 \leq t^{*}<\bar{t}<\widehat{t}<1$ such that for all $t \in\left(t^{*}, \hat{t}\right)$, we have

$$
\begin{gathered}
f\left(\gamma_{x, y}(t)\right)>f(y), \quad \bar{b}(x, y, t)>0, \\
f\left(\gamma_{x, y}\left(t^{*}\right)\right)=f(y) .
\end{gathered}
$$


Now, by the Mean Value Theorem, there exists $t_{0} \in\left(t^{*}, \bar{t}\right)$ such that

$$
\begin{aligned}
f\left(\gamma_{x, y}(\bar{t})\right)-f(y) & =f\left(\gamma_{x, y}(\bar{t})\right)-f\left(\gamma_{x, y}\left(t^{*}\right)\right) \\
& =\left(\bar{t}-t^{*}\right) d f_{\bar{x}} \gamma_{x, y}^{\prime}\left(t_{0}\right),
\end{aligned}
$$

where $\widehat{x}=\gamma_{x, y}\left(t_{0}\right)$. The left-hand side is positive by our hypothesis, but the right-hand size is zero by (56), as $\widehat{x} \epsilon$ $\Omega^{\prime \prime}$, and hence, we have a contradiction. This completes the proof.

We can also easily obtain the following results.

Theorem 25. Let $M$ be a Riemannian manifold, let $S \subseteq M$ be an open geodesic invex set with respect to $\eta$, and let $f$ : $S \rightarrow R$ be differentiable GPBPIX with respect to $\eta$ and $b$. Then $f$ is GPBIX with respect to $\eta$ and $\bar{b}$, where $\bar{b}(x, y)=$ $\lim _{t \rightarrow 0^{+}} b(x, y, t)$.

Theorem 26. Let $M$ be a Riemannian manifold, let $S \subseteq M$ be an open geodesic invex set with respect to $\eta$, and let $f$ : $S \rightarrow R$ be differentiable GPBPIX with respect to $\eta$ and $b$. Then $f$ is GQBIX with respect to $\eta$ and $\bar{b}$, where $\bar{b}(x, y)=$ $\lim _{t \rightarrow 0^{+}} b(x, y, t)$.

Remark 27. Theorems 25 and 26 generalize the known results from $R^{n}$ to Riemannian manifolds.

\section{Optimality Conditions and Duality}

In this section, we discuss a multiobjective optimization problem (VOP) involving generalized GBIX functions and obtain the Kuhn-Tucker sufficient conditions for a feasible point $x^{0}$ of (VOP) to be an efficient or properly efficient solution. We also formulate a Mond-Weir type dual for (VOP) and give various types of duality results. All these conclusions extend the corresponding results on $R^{n}$ (see, e.g., $[2,4,5,7,8]$ and the references therein) to Riemannian manifolds under the assumptions of GBIX functions and their generalization introduced in Section 2.

Let $M$ be a Riemannian manifold and let $S \subseteq M$ be an open invex set with respect to $\eta: M \times M \rightarrow T M$. We are concerned with the following multiple objective optimization problem:

$$
\begin{array}{ll}
(\mathrm{VOP}) \quad \min & f(x)=\left(f_{1}(x), \ldots, f_{k}(x)\right), \\
\text { s.t. } & g(x) \leqq 0
\end{array}
$$

where $f: S \rightarrow R^{k}$ and $g: S \rightarrow R^{m}$ are differentiable functions. Let $D=\left\{x \mid x \in S, g_{i}(x) \leqq 0, i=1,2, \ldots, m\right\}$ be the set of feasible solutions for (VOP), and $I\left(x^{0}\right)=\{i \mid$ $\left.g_{i}\left(x^{0}\right)=0\right\}$.
For vector inequalities we adopt the usual notions. If $x, y \in R^{m}$, then

$$
\begin{gathered}
x=y \Longleftrightarrow x_{i}=y_{i}, \quad i=1,2, \ldots, m, \\
x<y \Longleftrightarrow x_{i}<y_{i}, \quad i=1,2, \ldots, m . \\
x \leqq y \Longleftrightarrow x_{i} \leqq y_{i}, \quad i=1,2, \ldots, m, \\
x \leq y \Longleftrightarrow x_{i} \leqq y_{i}, \quad i=1,2, \ldots, m, \quad x \neq y, \\
x \notin y \quad \text { is the negation of } x \leq y .
\end{gathered}
$$

Definition 28 (see [34]). A feasible point $x^{0} \in D$ is said to be an efficient solution of (VOP) if there exists no other feasible point $x \in D$ such that $f(x) \leq f\left(x^{0}\right)$.

Definition 29 (see [34]). The point $x^{0}$ is said to be properly efficient of (VOP) if it is efficient for (VOP) and if there exists a scalar $M>0$ such that, for each $i$,

$$
\frac{f_{i}\left(x^{0}\right)-f_{i}(x)}{f_{j}(x)-f_{j}\left(x^{0}\right)} \leqq M
$$

for some $j$ such that $f_{j}(x)>f_{j}\left(x^{0}\right)$, whenever $x$ is feasible for (VOP) and $f_{i}(x)<f_{i}\left(x^{0}\right)$.

Theorem 30. Let $x^{0}$ be a feasible solution to (VOP). Assume that for every feasible point $x$, there exist scalars $\lambda_{j}>0, j=$ $1,2, \ldots, k$ and $\mu_{i} \geqq 0, i \in I\left(x^{0}\right)$, such that

$$
\sum_{j=1}^{k} \lambda_{j} d\left(f_{j}\right)_{x^{0}} \eta\left(x, x^{0}\right)+\sum_{i \in I\left(x^{0}\right)} \mu_{i} d\left(g_{i}\right)_{x^{0}} \eta\left(x, x^{0}\right) \geqq 0 .
$$

Suppose that $f_{j}, j=1,2, \ldots, k$ are GBIX with respect to $\eta$ and $b$ at $x^{0}$, and $g_{i}, i \in I\left(x^{0}\right)$ is GBIX with respect to $\eta$ and $\bar{b}$ at $x^{0}$. If $b\left(x, x^{0}\right)>0$ for any $x \in D$, then $x^{0}$ is a properly efficient solution for (VOP).

Proof. Since $f_{j}, j=1,2, \ldots, k$ and $g_{i}, i \in I\left(x^{0}\right)$ are GBIX, from condition (62), we get

$$
\begin{aligned}
b(x, & \left.x^{0}\right) \sum_{j=1}^{k} \lambda_{j}\left[f_{j}(x)-f_{j}\left(x^{0}\right)\right] \\
& \geqq \sum_{j=1}^{k} \lambda_{j} d\left(f_{j}\right)_{x^{0}} \eta\left(x, x^{0}\right) \\
& \geqq-\sum_{i \in I\left(x^{0}\right)} \mu_{i} d\left(g_{i}\right)_{x^{0}} \eta\left(x, x^{0}\right) \\
& \geqq \bar{b}\left(x, x^{0}\right)\left[\sum_{i \in I\left(x^{0}\right)} \mu_{i} g_{i}\left(x^{0}\right)-\sum_{i \in I\left(x^{0}\right)} \mu_{i} g_{i}(x)\right] \\
& =-\bar{b}\left(x, x^{0}\right) \sum_{i \in I\left(x^{0}\right)} \mu_{i} g_{i}(x)
\end{aligned}
$$$$
\geqq 0 \text {. }
$$ 
Thus,

$$
\begin{aligned}
& b\left(x, x^{0}\right) \sum_{j=1}^{k} \lambda_{j} f_{j}(x) \\
& \geqq b\left(x, x^{0}\right) \sum_{j=1}^{k} \lambda_{j} f_{j}\left(x^{0}\right), \quad \forall x \in D,
\end{aligned}
$$

which implies that

$$
x^{0} \text { minimize } \sum_{j=1}^{k} \omega_{j} f_{j}(x), \quad \text { subject to } g(x) \leqq 0,
$$

where

$$
\omega_{j}=\frac{\lambda_{j}}{b\left(x, x^{0}\right) \sum_{j=1}^{k} \lambda_{j}} .
$$

Hence, from Theorem 4.11 of [35], $x^{0}$ is a properly efficient solution for (VOP). This completes the proof.

Remark 31. Theorem 30 is a generalization of Theorem 5.5 in [23].

Theorem 32. Let $x^{0}$ be a feasible solution for (VOP). If there exist scalars $\lambda_{j} \geqq 0, j=1,2, \ldots, k, \sum_{j=1}^{k} \lambda_{j}=1, \mu_{i} \geqq$ $0, i \in I\left(x^{0}\right)$, such that the triplet $\left(x^{0}, \lambda_{j}, \mu_{i}\right)$ satisfies (62) in Theorem 30. Assume that $\sum_{j=1}^{k} \lambda_{j} f_{j}$ is strictly GBIX with respect to $\eta$ and $b$ at $x^{0}$, and $g_{i}, i \in I\left(x^{0}\right)$ is GBIX with respect to $\eta$ and $\bar{b}$ at $x^{0}$. Then $x^{0}$ is an efficient solution for problem (VOP).

Proof. Suppose that $x^{0}$ is not an efficient solution for (VOP). Then there exists a feasible point $x \in D$ such that

$$
f(x) \leq f\left(x^{0}\right)
$$

Since $\sum_{j=1}^{k} \lambda_{j} f_{j}(x)$ is strictly GBIX, we conclude

$$
\begin{gathered}
b\left(x, x^{0}\right)\left[\sum_{j=1}^{k} \lambda_{j} f_{j}(x)-\sum_{j=1}^{k} \lambda_{j} f_{j}\left(x^{0}\right)\right] \leqq 0 \\
\Longrightarrow \sum_{j=1}^{k} \lambda_{j} d\left(f_{j}\right)_{x^{0}} \eta\left(x, x^{0}\right)<0 .
\end{gathered}
$$

Also, the GBIX of $g_{i}, i \in I\left(x^{0}\right)$ yields

$$
\begin{gathered}
\bar{b}\left(x, x^{0}\right)\left[\sum_{i \in I\left(x^{0}\right)} \mu_{i} g_{i}(x)-\sum_{i \in I\left(x^{0}\right)} \mu_{i} g_{0}\left(x^{0}\right)\right] \leqq 0 \\
\Longrightarrow \sum_{i \in I\left(x^{0}\right)} \mu_{i} d\left(g_{i}\right)_{x^{0}} \eta\left(x, x^{0}\right) \leqq 0 .
\end{gathered}
$$

Adding (68) and (69), we obtain a contradiction to (62). This completes the proof.
Remark 33. Proceeding along the same lines as in Theorem 30, it can be easily seen that $x^{0}$ becomes properly efficient to (VOP) in the above theorem, if $\lambda_{j}>0$, for all $j=1,2, \ldots, k$.

Theorem 34. Suppose that there exist a feasible point $x^{0}$ and scalars $\lambda_{j}>0, j=1,2, \ldots, k \mu_{i} \geqq 0, i \in I\left(x^{0}\right)$, such that (62) of Theorem 30 holds. Let $\sum_{j=1}^{k} \lambda_{j} f_{j}$ be GPBIX with respect to $\eta$ and $b$ at $x^{0}$ and let $g_{i}, i \in I\left(x^{0}\right)$ be GQBIX with respect to $\eta$ and $\bar{b}$ at $x^{0}$. If $b\left(x, x^{0}\right)>0$ and $\bar{b}\left(x, x^{0}\right)>0$ for any $x \in D$, then $x^{0}$ is a properly efficient solution for (VOP).

Proof. Since $g_{i}(x) \leqq g_{i}\left(x^{0}\right)=0, i \in I\left(x^{0}\right)$, and $g_{i}, i \in I\left(x^{0}\right)$ are GQBIX functions, we obtain

$$
\bar{b}\left(x, x^{0}\right) \sum_{i \in I\left(x^{0}\right)} \mu_{i} d\left(g_{i}\right)_{x^{0}} \eta\left(x, x^{0}\right) \leqq 0, \quad \forall x \in D,
$$

which along with (62) yields

$$
d\left(\sum_{j=1}^{k} f_{j}\right)_{x^{0}} \eta\left(x, x^{0}\right) \geqq 0
$$

Since $\sum_{j=1}^{k} \lambda_{j} f_{j}$ is GPBIX, the above inequality implies that

$$
b\left(x, x^{0}\right) \sum_{j=1}^{k} \lambda_{j} f_{j}(x) \geqq b\left(x, x^{0}\right) \sum_{j=1}^{k} \lambda_{j} f_{j}\left(x^{0}\right) .
$$

Thus, we conclude that $x^{0} \operatorname{minimize} \sum_{j=1}^{k} \omega_{j} f_{j}(x)$, under the constraint $g(x) \leqq 0$, where

$$
\omega_{j}=\frac{\lambda_{j}}{b\left(x, x^{0}\right) \sum_{j=1}^{k} \lambda_{j}} .
$$

Therefore, $x^{0}$ is a properly efficient for (VOP). This completes the proof.

Theorem 35. Let $x^{0}$ be a feasible point for (VOP). Assume that there exist scalars $\lambda_{j} \geqq 0, j=1,2, \ldots, k, \sum_{j=1}^{k} \lambda_{j}=1, \mu_{i} \geqq 0$, $i \in I\left(x^{0}\right)$, such that (62) of Theorem 30 holds. Let $\sum_{j=1}^{k} \lambda_{j} f_{j}$ be strictly GPBIX with respect to $\eta$ and $b$ at $x^{0}$, and let $g_{i}, i \in I\left(x^{0}\right)$ be GQBIX with respect to $\eta$ and $\bar{b}$ at $x^{0}$. If $\bar{b}\left(x, x^{0}\right)>0$ for any $x \in D$, then $x^{0}$ is an efficient solution for problem (VOP).

Proof. Suppose that $x^{0}$ is not efficient for (VOP). Then, there exists a feasible $x$ of (VOP) such that

$$
f(x) \leq f\left(x^{0}\right)
$$

which yields

$$
b\left(x, x^{0}\right) \sum_{j=1}^{k} \lambda_{j} f_{j}(x) \leqq b\left(x, x^{0}\right) \sum_{j=1}^{k} \lambda_{j} f_{j}\left(x^{0}\right) .
$$


It follows from the strict GPBIX of $\sum_{j=1}^{k} \lambda_{j} f_{j}$ that

$$
\sum_{j=1}^{k} \lambda_{j} d\left(f_{j}\right)_{x^{0}} \eta\left(x, x^{0}\right)=d\left(\sum_{j=1}^{k} \lambda_{j} f_{j}\right)_{x^{0}} \eta\left(x, x^{0}\right)<0 .
$$

Also, from the GQBIX of $g_{i}, i \in I\left(x^{0}\right)$, we conclude

$$
\begin{aligned}
& \sum_{i \in I\left(x^{0}\right)} \mu_{i} g_{i}(x) \leqq \sum_{i \in I\left(x^{0}\right)} \mu_{i} g_{i}\left(x^{0}\right), \\
& \Longrightarrow \bar{b}\left(x, x^{0}\right) \sum_{i \in I\left(x^{0}\right)} \mu_{i} d\left(g_{i}\right)_{x^{0}} \eta\left(x, x^{0}\right) \leqq 0, \quad \forall x \in D .
\end{aligned}
$$

The proof now is similar to the proof of Theorem 32. This completes the proof.

Remark 36. Similarly as in Theorem 34, it can be easily seen that $x^{0}$ becomes properly efficient for (VOP) in the above theorem if $\lambda_{j}>0$ for all $j=1,2, \ldots, k$.

Remark 37. In Theorems 34 and 35, the results still hold when $g_{i}$ is replaced by $\mu_{i} g_{i}$ with $i \in I\left(x^{0}\right)$.

We now consider the following Mond-Weir vector dual of (VOP):

$$
\begin{aligned}
& \text { (MVD) } \max f(u) \text {, } \\
& \text { s.t. } \sum_{j=1}^{k} \lambda_{j} d\left(f_{j}\right)_{u} \eta(x, u) \\
& +\sum_{i=1}^{m} \mu_{i} d\left(g_{i}\right)_{u} \eta(x, u)=0, \\
& \sum_{i=1}^{m} \mu_{i} g_{i}(u) \geqq 0, \\
& \mu_{i} \geqq 0, \quad i=1,2, \ldots, m, \\
& \lambda_{j} \geqq 0, \quad j=1,2, \ldots, k, \quad \sum_{j=1}^{k} \lambda_{j}=1,
\end{aligned}
$$

for all $x \in D$, and $f$ and $g$ are differentiable functions on $S \subseteq M$.

We now prove various duality results for (VOP) and (MVD).

Theorem 38 (weak duality). Let $x$ and $(u, \lambda, \mu)$ be feasible for (VOP) and (MVD), respectively. If also either

(a) $\lambda_{j}>0$ for all $j=1,2, \ldots, k$ and $\sum_{j=1}^{k} \lambda_{j} f_{j}$ is GPBIX with respect to $\eta$ and $b$ at $u, \sum_{i=1}^{m} \mu_{i} g_{i}$ is GQBIX with respect to $\eta$ and $\bar{b}$ at $u$, and $b(x, u)>0, \bar{b}(x, u)>0$, or

(b) $\sum_{j=1}^{k} \lambda_{j} f_{j}$ is strictly GPBIX with respect to $\eta$ and $b$ at $u, \sum_{i=1}^{m} \mu_{i} g_{i}$ is GQBIX with respect to $\eta$ and $\bar{b}$ at $u$, and $\bar{b}(x, u)>0$,

then $f(x) \nless \leq f(u)$.
Proof. We proceed by contradiction. If $f(x) \leq f(u)$, then for $\lambda_{j}>0, j=1,2, \ldots, k$ and $b>0$, we get

$$
b(x, u) \sum_{j=1}^{k} \lambda_{j} f_{j}(x)<b(x, u) \sum_{j=1}^{k} \lambda_{j} f_{j}(u),
$$

or for $\lambda_{j} \geqq 0, j=1,2, \ldots, k, \sum_{i=1}^{k} \lambda_{j}=1$, we have

$$
b(x, u) \sum_{j=1}^{k} \lambda_{j} f_{j}(x) \leqq b(x, u) \sum_{j=1}^{k} \lambda_{j} f_{j}(u) .
$$

Now since $\sum_{j=1}^{k} \lambda_{j} f_{j}$ is GPBIX or strictly GPBIX, the above two inequalities both give

$$
\sum_{j=1}^{k} \lambda_{j} d\left(f_{j}\right)_{u} \eta(x, u)<0, \quad \forall x \in D .
$$

It follows from (79) that

$$
\sum_{i=1}^{m} \mu_{i} g_{i}(x) \leqq 0 \leqq \sum_{i=1}^{m} \mu_{i} g_{i}(u), \quad \forall x \in D,
$$

and the GQBIX of $\sum_{i=1}^{m} \mu_{i} g_{i}$ gives

$$
\begin{aligned}
\bar{b}(x, u) d\left(\sum_{i=1}^{m} \mu_{i} g_{i}\right)_{u} \eta(x, u) \\
=\bar{b}(x, u) \sum_{i=1}^{m} \mu_{i} d\left(g_{i}\right)_{u} \eta(x, u) \leqq 0 .
\end{aligned}
$$

By adding (84) and (86), we obtain a contradiction to (78). This completes the proof.

Theorem 39 (strong duality). Let $x^{0}$ be an efficient solution for (VOP) at which the Kuhn-Tucker conditions are satisfied. If for all feasible solutions $(u, \lambda, \mu)$ of (MVD) $\sum_{j=1}^{k} \lambda_{j} f_{j}$ is strictly GPBIX with respect to $\eta$ and $b$ and $\sum_{i=1}^{k} \mu_{i} g_{i}$ is GQBIX with respect to $\eta$ and $\bar{b}$, where $\bar{b}(x, u)>0$ for all $x \in D$, then there exists $\left(x^{0}, \mu^{0}\right) \in R^{k} \times R^{m}$ such that $\left(x^{0}, \lambda^{0}, \mu^{0}\right)$ is efficient for (MVD) and the objective function values of (VOP) and (MVD) are equal.

Proof. The assumption in the above theorem implies that there exist scalars $\lambda_{j}^{0} \geqq 0, j=1,2, \ldots, k, \sum_{j=1}^{k} \lambda_{j}=1$, and $\mu_{i}^{0} \geqq 0, i=1,2, \ldots, m$ such that the Kuhn-Tucker conditions hold:

$$
\begin{gathered}
\sum_{j=1}^{k} \lambda_{j}^{0} d\left(f_{j}\right)_{x^{0}} \eta\left(x, x^{0}\right)+\sum_{i=1}^{m} \mu_{i}^{0} d\left(g_{i}\right)_{x^{0}} \eta\left(x, x^{0}\right)=0, \\
\sum_{i=1}^{m} \mu_{i}^{0} g_{i}\left(x^{0}\right)=0,
\end{gathered}
$$

which gives that the triplet $\left(x^{0}, \lambda^{0}, \mu^{0}\right)$ is feasible for (MVD). If $\left(x^{0}, \lambda^{0}, \mu^{0}\right)$ is not efficient, then there exists a feasible $(u, \lambda, \mu)$ for (MVD) such that $f\left(x^{0}\right) \leq f(u)$, which contradicts the weak duality. This completes the proof. 
Theorem 40. Let $x^{0}$ be feasible for (VOP), and let $\left(u^{0}, \lambda^{0}, \mu^{0}\right)$ be feasible for (MVD) such that

$$
\sum_{j=1}^{k} \lambda_{j}^{0} f_{j}\left(x^{0}\right)=\sum_{j=1}^{k} \lambda_{j}^{0} f_{j}\left(u^{0}\right) .
$$

Suppose that $\sum_{j=1}^{k} \lambda_{j}^{0} f_{j}$ is strictly GPBIX at $u^{0}$ with respect to $\eta$ and $b$, and $\sum_{i=1}^{m} \mu_{i}^{0} g_{i}$ is GQBIX at $u^{0}$ with respect to $\eta$ and $\bar{b}$. If $b\left(x^{0}, u^{0}\right)>0$ and $\bar{b}\left(x^{0}, u^{0}\right)>0$, then $x^{0}=u^{0}$.

Proof. Let $x^{0} \neq u^{0}$. Since $\sum_{i=1}^{m} \mu_{i}^{0} g_{i}$ is GQBIX and $\left(u^{0}, \lambda^{0}, \mu^{0}\right)$ is feasible for (MVD), we conclude

$$
\begin{aligned}
\sum_{i=1}^{m} \mu_{i}^{0} g_{i}\left(x^{0}\right) \leqq & \sum_{i=1}^{m} \mu_{i}^{0} g_{i}\left(u^{0}\right) \Longrightarrow \bar{b}\left(x^{0}, u^{0}\right) \\
& \times \sum_{i=1}^{m} \mu_{i}^{0} d\left(g_{i}\right)_{u^{0}} \eta\left(x^{0}, u^{0}\right) \leqq 0 .
\end{aligned}
$$

It follows from (78) that

$$
\sum_{j=1}^{k} \lambda_{j}^{0} d\left(f_{j}\right)_{u^{0}} \eta\left(x^{0}, u^{0}\right) \geqq 0 .
$$

Again from the strict GPBIX of $\sum_{j=1}^{k} \lambda_{j} f_{j}$, we have

$$
b\left(x^{0}, u^{0}\right) \sum_{j=1}^{k} \lambda_{j}^{0} f_{j}\left(x^{0}\right)>b\left(x^{0}, u^{0}\right) \sum_{j=1}^{k} \lambda_{j}^{0} f\left(u^{0}\right) .
$$

This is a contradiction. Therefore, $x^{0}=u^{0}$. This completes the proof.

Theorem 41. Suppose that there exist a feasible $x^{0}$ for (VOP) and a feasible $\left(u^{0}, \lambda^{0}, \mu^{0}\right)$ for (MVD) such that

$$
f_{j}\left(x^{0}\right)=f_{j}\left(u^{0}\right), \quad j=1,2, \ldots, k .
$$

Iffor $j=1,2, \ldots, k, \lambda_{j}>0$ and

$$
\frac{\sum_{j=1}^{k} \lambda_{j}^{0} f_{j}}{\sum_{i=1}^{m} \mu_{i}^{0} g_{i}}
$$

is GPBIX/GQBIX with respect to $\eta$ and $b / \bar{b}$ at $u^{0}$, then $x^{0}$ is properly efficient for (VOP). Also if for each feasible $(u, \lambda, \mu)$ of (MVD),

$$
\frac{\sum_{j=1}^{k} \lambda_{j} f_{j}}{\sum_{i=1}^{m} \mu_{i} g_{i}}
$$

is GPBIX/GQBIX with respect to $\eta$ and $b / \bar{b}$ and $b\left(x^{0}, u^{0}\right)>0$, $\bar{b}\left(x^{0}, u^{0}\right)>0$, then $u^{0}$ is properly efficient of (MVD).

Proof. Suppose that $x^{0}$ is not an efficient solution for (VOP). Then there exists a feasible $x$ for (VOP) such that

$$
f(x) \leq f\left(x^{0}\right) .
$$

Using condition (92), we contradict the weak duality. Thus, $x^{0}$ is efficient for (VOP). If $x^{0}$ is not properly efficient for (VOP), then there exist a feasible $x$ and an index $i$ such that

$$
f_{i}\left(x^{0}\right)-f_{i}(x)>M\left(f_{j}(x)-f_{j}\left(x^{0}\right)\right),
$$

for all $M>0$ and all $j$ such that $f_{j}(x)>f_{j}\left(x^{0}\right)$ whenever $f_{i}(x)<f_{i}\left(x^{0}\right)$. Again utilizing condition (92), we obtain

$$
f_{i}(x)-f_{i}\left(u^{0}\right)<-M\left(f_{j}(x)-f_{j}\left(u^{0}\right)\right)
$$

for all $M>0$ and all $j$ such that

$$
f_{j}(x)>f_{j}\left(u^{0}\right)
$$

whenever

$$
f_{i}(x)<f_{i}\left(u^{0}\right)
$$

Since $M$ can be made large, hence for $\lambda_{j}^{0}>0$, we get the inequality

$$
\sum_{j=1}^{k} \lambda_{j}^{0}\left(f_{j}(x)-f_{j}\left(u^{0}\right)\right)<0
$$

which contradicts the weak duality. Therefore, $x^{0}$ is properly efficient for (VOP).

To prove the second half of this theorem, let us assume on the contrary that $\left(u^{0}, \lambda^{0}, \mu^{0}\right)$ is not an efficient solution of (MVD). Then there exists a feasible point $(u, \lambda, \mu)$ of (MVD) such that

$$
f\left(u^{0}\right) \leq f(u)
$$

By applying condition (92), we get a contradiction to weak duality. Hence, $\left(u^{0}, \lambda^{0}, \mu^{0}\right)$ is an efficient solution of (MVD).

If $\left(u^{0}, \lambda^{0}, \mu^{0}\right)$ is not properly efficient for (MVD), then there exist a feasible $(u, \lambda, \mu)$ of (MVD) and an index $i$ such that

$$
f_{i}(u)-f_{i}\left(u^{0}\right)>M\left(f_{j}\left(u^{0}\right)-f_{j}(u)\right)
$$

for all $M>0$ and for all $j$ such that

$$
f_{j}\left(u^{0}\right)>f_{j}(u)
$$

whenever

$$
f_{i}\left(u^{0}\right)<f_{i}(u)
$$

Utilizing (92) again, we obtain

$$
f_{i}(u)-f_{i}\left(x^{0}\right)>M\left(f_{j}\left(x^{0}\right)-f_{j}(u)\right),
$$

for all $M>0$ and for all $j$ such that

$$
f_{j}\left(x^{0}\right)>f_{j}(u),
$$


whenever

$$
f_{i}\left(x^{0}\right)<f_{i}(u) .
$$

Now using the same argument as in the first part of the theorem, we get

$$
\sum_{j=1}^{k} \lambda_{j}^{0}\left(f_{j}\left(x^{0}\right)-f_{j}(u)\right)<0,
$$

which contradicts the weak duality. This completes the proof.

\section{Conflict of Interests}

The authors declare that there is no conflict of interests regarding the publication of this paper.

\section{Acknowledgments}

The authors are grateful to the editor and the referees for their valuable comments and suggestions. This work was supported by the Key Program of NSFC (Grant no. 70831005) and the National Natural Science Foundation of China (11171237).

\section{References}

[1] C. R. Bector and C. Singh, "B-vex functions," Journal of Optimization Theory and Applications, vol. 71, no. 2, pp. 237-253, 1991.

[2] M. A. Hanson, "On sufficiency of the Kuhn-Tucker conditions," Journal of Mathematical Analysis and Applications, vol. 80, no. 2, pp. 545-550, 1981.

[3] A. Ben-Israel and B. Mond, "What is invexity?" Australian Mathematical Society B, vol. 28, no. 1, pp. 1-9, 1986.

[4] T. Weir and B. Mond, "Pre-invex functions in multiple objective optimization," Journal of Mathematical Analysis and Applications, vol. 136, no. 1, pp. 29-38, 1988.

[5] C. R. Bector, S. K. Suneja, and C. S. Lalitha, "Generalized Bvex functions and generalized $B$-vex programming," Journal of Optimization Theory and Applications, vol. 76, no. 3, pp. 561576, 1993.

[6] S. K. Suneja, C. Singh, and C. R. Bector, "Generalization of preinvex and B-vex functions," Journal of Optimization Theory and Applications, vol. 76, no. 3, pp. 577-587, 1993.

[7] R. N. Kaul, S. K. Suneja, and M. K. Srivastava, "Optimality criteria and duality in multiple-objective optimization involving generalized invexity," Journal of Optimization Theory and Applications, vol. 80, no. 3, pp. 465-482, 1994.

[8] R. Pini and C. Singh, "A survey of recent [1985-1995] advances in generalized convexity with applications to duality theory and optimality conditions," Optimization, vol. 39, no. 4, pp. 311-360, 1997.

[9] T. Morsy, A Study on Generalized Convex Mathematical Programming Problems [M.S. thesis], Faculty of Science, Suez Canal University, Ismailia, Egypt, 2003.

[10] C. J. Goh and X. Q. Yang, Duality in Optimization and Variational Inequalites, Taylor \& Francis, London, UK, 2002.
[11] M. Arana-Jiménez, G. Ruiz-Garzón, R. Osuna-Gómez, and B. Hernández-Jiménez, "Duality and a characterization of pseudoinvexity for Pareto and weak Pareto solutions in nondifferentiable multiobjective programming," Journal of Optimization Theory and Applications, vol. 156, no. 2, pp. 266-277, 2013.

[12] S. R. Mohan and S. K. Neogy, "On invex sets and preinvex functions," Journal of Mathematical Analysis and Applications, vol. 189, no. 3, pp. 901-908, 1995.

[13] C. Li, B. S. Mordukhovich, J. Wang, and J.-C. Yao, "Weak sharp minima on Riemannian manifolds," SIAM Journal on Optimization, vol. 21, no. 4, pp. 1523-1560, 2011.

[14] R. Bartolo, A. Germinario, and M. Sánchez, "Convexity of domains of Riemannian manifolds," Annals of Global Analysis and Geometry, vol. 21, no. 1, pp. 63-83, 2002.

[15] M. Ferrara and S. Mititelu, "Mond-Weir duality in vector programming with generalized invex functions on differentiable manifolds," Balkan Journal of Geometry and its Applications, vol. 11, no. 2, pp. 80-87, 2006.

[16] C. Udrişte, "Kuhn-Tucker theorem on Riemannian manifolds," in Topics in a Differential Geometry, Colloquia Mathematica Societatis János Bolyai, pp. 1247-1259, North-Holland, Debrecen, Hungary, 1984.

[17] A. Barani and M. R. Pouryayevali, "Invex sets and preinvex functions on Riemannian manifolds," Journal of Mathematical Analysis and Applications, vol. 328, no. 2, pp. 767-779, 2007.

[18] A. Barani and M. R. Pouryayevali, "Invariant monotone vector fields on Riemannian manifolds," Nonlinear Analysis: Theory, Methods \& Applications, vol. 70, no. 5, pp. 1850-1861, 2009.

[19] A. Iqbal, I. Ahmad, and S. Ali, "Strong geodesic $\alpha$-preinvexity and invariant $\alpha$-monotonicity on Riemannian manifolds," Numerical Functional Analysis and Optimization, vol. 31, no. 12, pp. 1342-1361, 2010.

[20] R. P. Agarwal, I. Ahmad, A. Iqbal, and S. Ali, "Generalized invex sets and preinvex functions on Riemannian manifolds," Taiwanese Journal of Mathematics, vol. 16, no. 5, pp. 1719-1732, 2012.

[21] R. P. Agarwal, I. Ahmad, A. Iqbal, and S. Ali, "Geodesic G-invex sets and semistrictly geodesic $\eta$-preinvex functions," Optimization, vol. 61, no. 9, pp. 1169-1174, 2012.

[22] R. Pini, "Convexity along curves and invexity," Optimization, vol. 29, no. 4, pp. 301-309, 1994.

[23] A. Barani and M. R. Pouryayevali, "Vector optimization problems under $d$-invexity on Riemannian manifolds," Differential Geometry, vol. 13, pp. 34-44, 2011.

[24] S. Mititelu, "Generalized invexity and vector optimization on differentiable manifolds," Differential Geometry, vol. 3, no. 1, pp. 21-31, 2001.

[25] D. Azagra, J. Ferrera, and F. López-Mesas, "Nonsmooth analysis and Hamilton-Jacobi equations on Riemannian manifolds," Journal of Functional Analysis, vol. 220, no. 2, pp. 304-361, 2005.

[26] L.-W. Zhou and N.-J. Huang, "Roughly geodesic $B$-invex and optimization problem on Hadamard manifolds," Taiwanese Journal of Mathematics, vol. 17, no. 3, pp. 833-855, 2013.

[27] T. Rapcsák, Smooth Nonlinear Optimization in $R^{n}$, Kluwer Academic, Dordrecht, The Netherlands, 1997.

[28] C. Udriste, "Convex functions and optimization methods on riemannian manifolds," in Mathematicsand Its Applications, vol. 297, Kluwer Academic, Boston, Mass, USA, 1994. 
[29] I. Chavel, Riemannian Geometry-A Modern Introduction, Cambridge University Press, Cambridge, UK, 1993.

[30] W. Klingenberg, Riemannian Geometry, vol. 1 of Walter de Gruyter Studies in Mathematics, Walter de Gruyter , Berlin, Germany, 1982.

[31] S. Lang, Fundamentals of Differential Geometry, vol. 191 of Graduate Texts in Mathematics, Springer, New York, NY, USA, 1999.

[32] Yu. S. Ledyaev and Q. J. Zhu, "Nonsmooth analysis on smooth manifolds," Transactions of the American Mathematical Society, vol. 359, no. 8, pp. 3687-3732, 2007.

[33] D. Azagra and J. Ferrera, "Proximal calculus on Riemannian manifolds," Mediterranean Journal of Mathematics, vol. 2, no. 4, pp. 437-450, 2005.

[34] A. M. Geoffrion, "Proper efficiency and the theory of vector maximization," Journal of Mathematical Analysis and Applications, vol. 22, pp. 618-630, 1968.

[35] V. Chankong and Y. Y. Haimes, Multiobjective Decision Making Theory and Methodology,, North-Holland, Amsterdam, The Netherlands, 1983. 


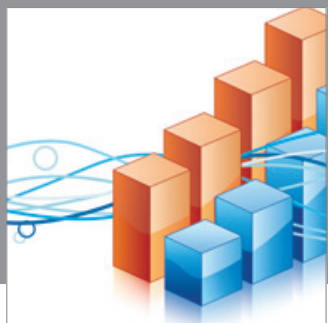

Advances in

Operations Research

mansans

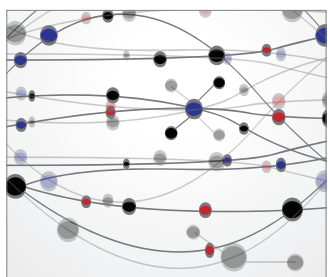

The Scientific World Journal
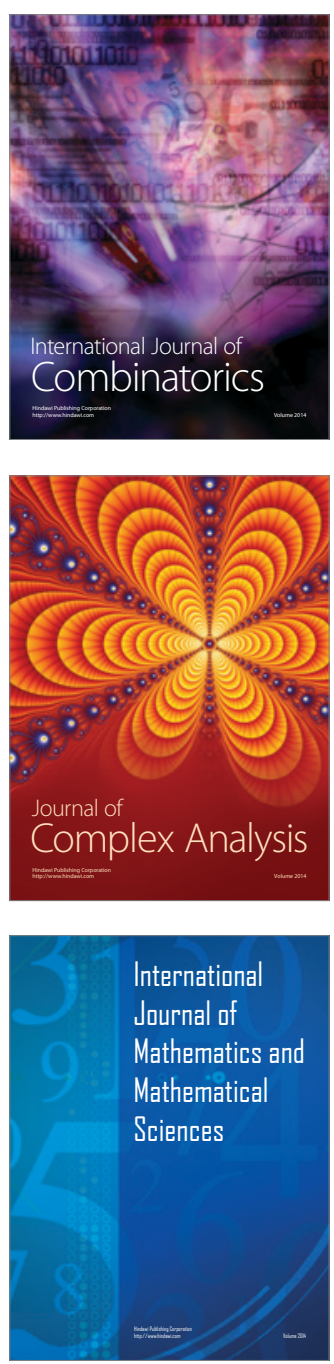
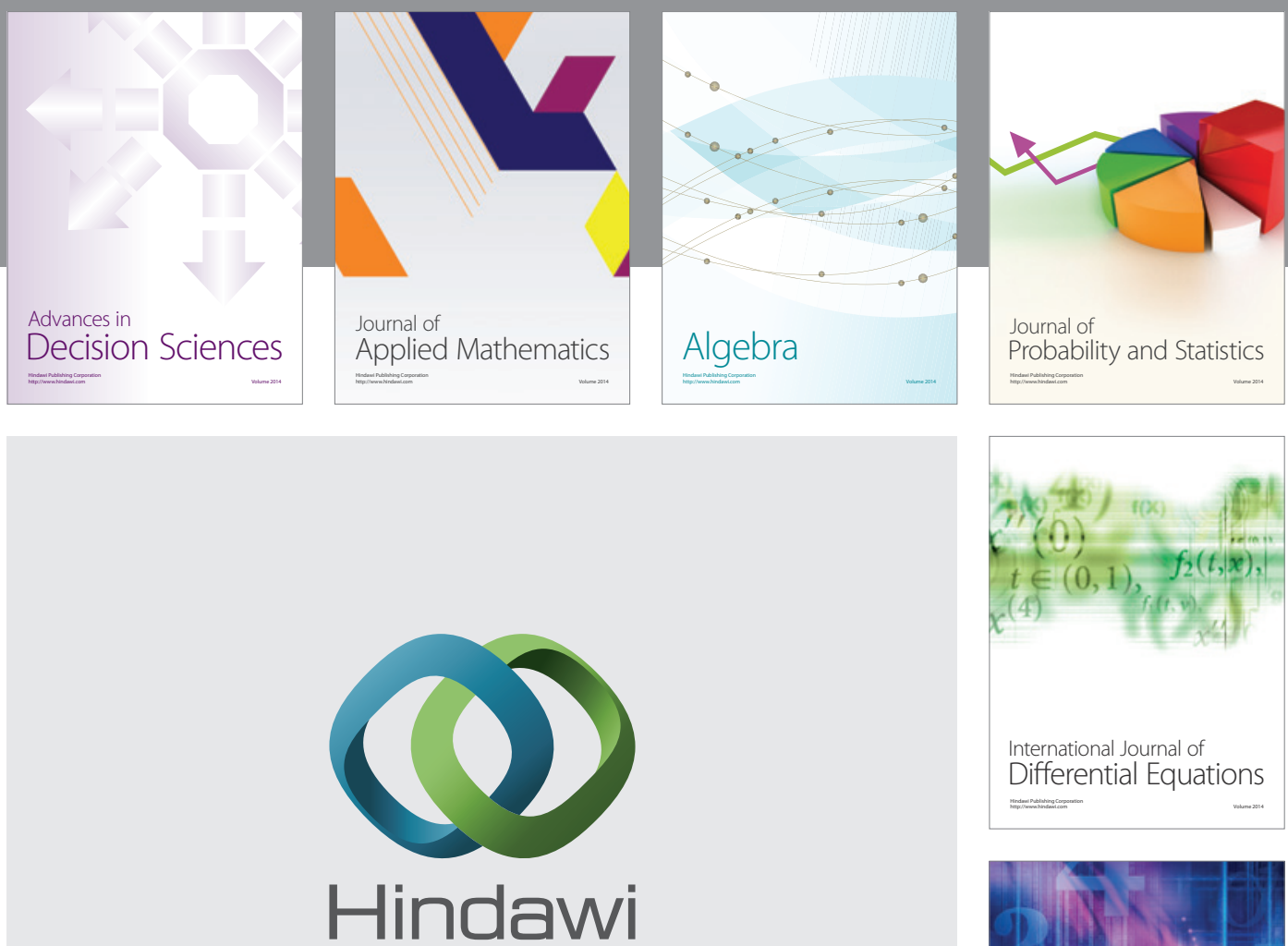

Submit your manuscripts at http://www.hindawi.com
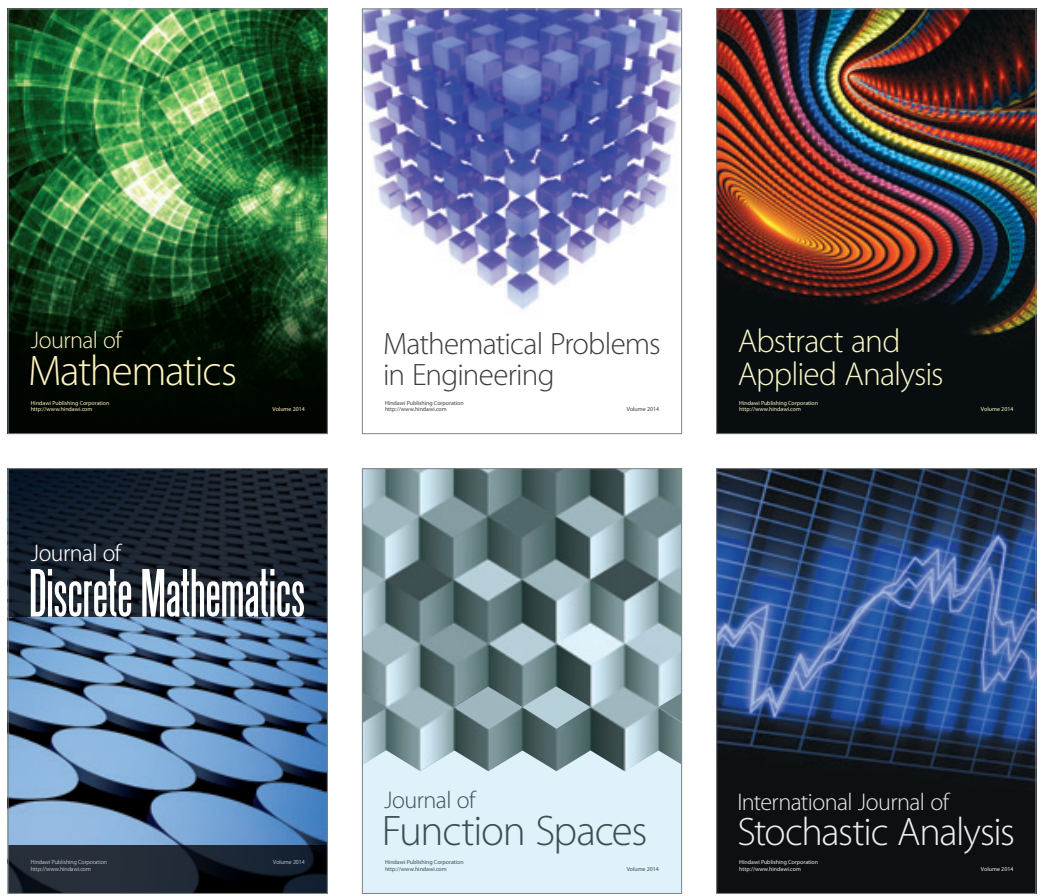

Journal of

Function Spaces

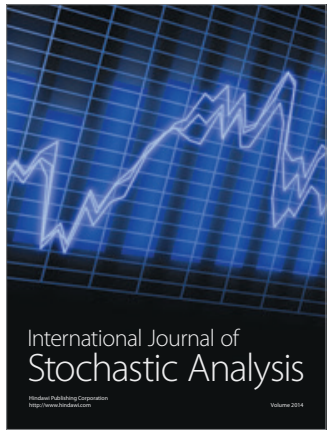

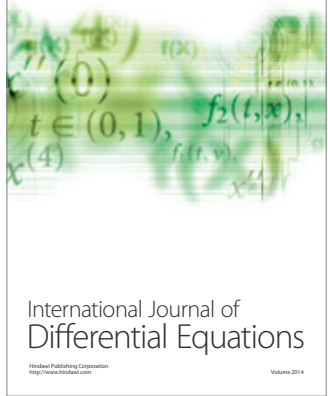
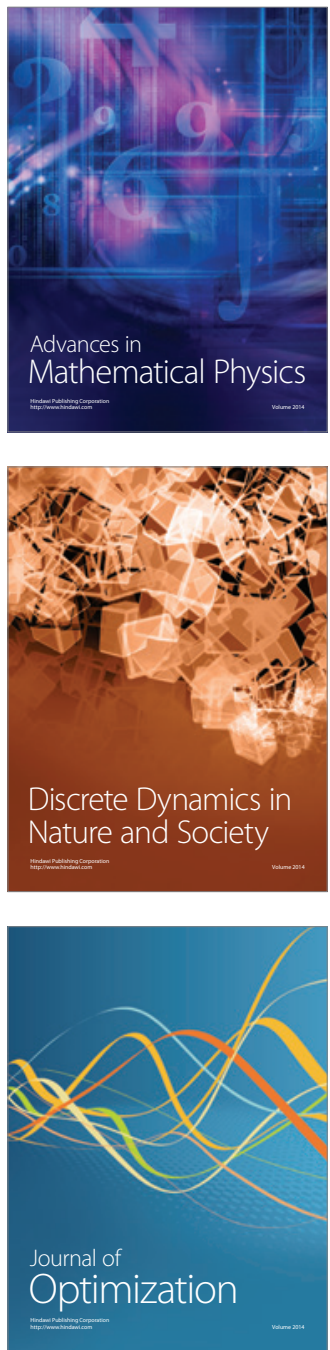